\title{
Robotics and Interactive Simulation
}

\author{
O. Khatib, O. Brock, K.-S. Chang, F. Conti, D. Ruspini, L. Sentis \\ Robotics Laboratory \\ Department of Computer Science \\ Stanford University, Stanford, California 94305 \\ email: \{khatib, oli, kcchang, ruspini, conti, lsentis\}@cs.stanford.edu
}

\begin{abstract}
A new field of robotics is emerging. Robots are today moving towards applications beyond the structured environment of a manufacturing plant. They are making their way into the everyday world that people inhabit. The article discusses models, strategies, and algorithms associated with the basic capabilities needed for robots to work, assist, and cooperate with humans. In addition to the capabilities they bring to the physical robot, these models and algorithms and more generally the body of developments in robotics is today making a significant impact on the virtual world. Haptic interaction with an accurate dynamic simulation provides unique insights into the real-world behaviors of physical systems. The potential applications of this emerging technology include virtual prototyping, animation, surgery, robotics, cooperative design, and education among many others.
\end{abstract}

\section{Introduction}

The successful introduction of robotics into human environments will rely on the development of competent and practical systems that are dependable, safe, and easy to use. To work, cooperate, assist, and interact with humans, the new generation of robot must have mechanical structures that accommodate the interaction with the human and adequately fit in his unstructured and sizable environment. Humancompatible robotic structures must integrate mobil- ity (legged or wheeled) and manipulation (preferably bi-manual), while providing the needed access to perception and monitoring (head camera) [5, 11]. Such diverse requirements can only be fulfilled by rather complex mechanisms, posing various challenges for algorithms in modeling, perception, programming, motion planning and control.

As advances are made in methodologies and techniques to address these challenges, it is becoming more and more apparent that their impact is going beyond the physical robot. Models and algorithms in robotics are providing the foundations for developments in many of the application areas found at the intersection of the physical and virtual worlds. These are areas where physical models are simulated and interacted with, such as virtual prototyping, haptics, molecular biology, training, games, collaborative work, and haptically augmented teleoperation $[4,9,10]$. A large number of ongoing efforts in robotics have resulted in significant advancements in these areas of application; in this article we will survey only some of the developments we have pursued in our laboratory that have contributed to this progress.

The new emerging applications in robotics share the requirement of simulating and controlling physical models with sufficient sophistication to recreate a complicated, physically consistent world and at sufficient speeds to allow user interaction. One example of such an application is the haptic display of virtual environments, where a robotic device permits the haptic interaction with a virtual environment. Applications for this technology include, among oth- 
ers, virtual prototyping, teleoperation, training, and games. The developments in this area are discussed in Section 2.

For haptic interaction to appear realistic to the user, the virtual object must exhibit the same simulated physical properties as the real object. These properties include the dynamics of rigid and articulated bodies and their mutual influences like those created by the impact forces during contact. To resolve the physical constraints arising in these situations and to simulate the dynamic behavior of complex objects in a cluttered environment, we have developed fast algorithms, which are presented in Section 3.

Both the virtual and the real world can be populated by complex, articulated and actuated mechanisms, like the humanoid robots mentioned earlier. Creating motions for these mechanisms with the purpose of performing a task, imitating a user command specified in a haptically simulated world, or reacting to interaction with other objects is a difficult task. In Section 4 our approach to whole-robot modeling and control is presented. It applies equally well to humanoid robots as to complex articulated bodies simulated in the virtual world.

In increasingly complex virtual and physical environments robots or objects can exhibit autonomous behavior: rather than being passively interacted with by a user, they themselves pursue an objective and can initiate interaction with other objects or the user. Section 5 discussed our approach to supporting the interactions resulting from independently and autonomously operating objects.

\section{Interactive Haptic Simula- tion}

A haptic device is a robot that, rather than manipulating the physical world in accordance with a user command, applies forces to a user's hand or finger in accordance with the forces resulting from the user's motion in a virtual environment. Following the input of a user, a virtual hand is moved in a virtual environment. As the virtual hand is subjected to forces
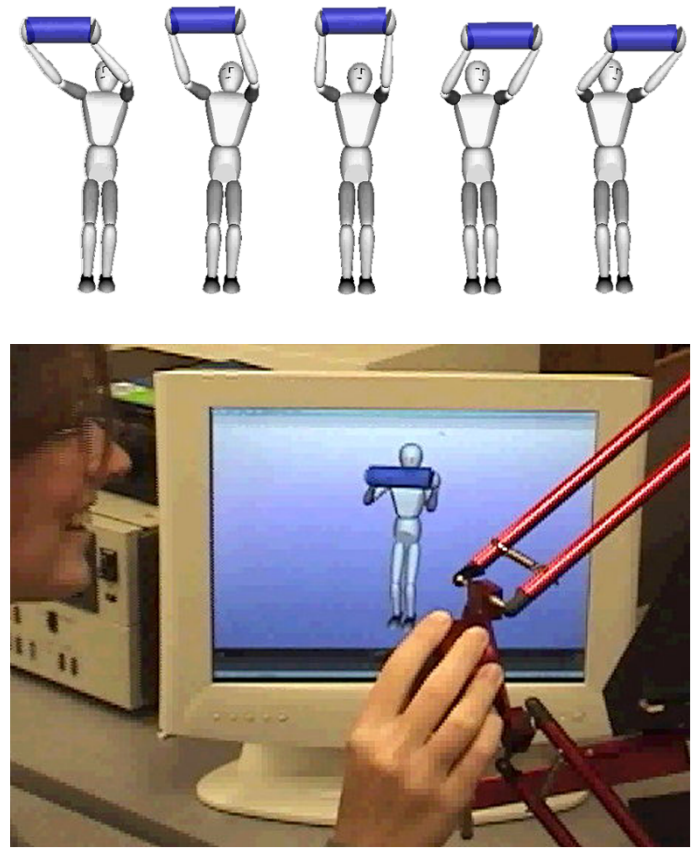

Figure 1: A user commands a virtual robot to move the manipulated object using a haptic device. The device permits the user to interact and control the manipulation task, while the humanoid robot autonomously adjusts its posture according to simple posture energies.

resulting from contact with objects, these forces are applied through the haptic device to the hand or finger of the user, enabling the haptic display of virtual environments.

A haptic device can also serve as an input device, as shown in Figure 1. The user specifies a motion for the object held by the humanoid robot. The sequence of images in Figure 1 (top) shows the resulting overall motion of the figure if the object is commanded to move from side to side. While the object is interactively manipulated, the figure autonomously adjusts its posture according to simple posture energies, as discussed in Section 4.

Such haptic displays of virtual worlds can be linked with the real world. This corresponds to transition- 
ing from haptically controlling and simulating a virtual environment to remotely controlling a physical environment. Such teleoperation with force feedback can be augmented with the simulated environment to produce a haptically augmented reality, where the world model is updated through monitoring and perception of the real world. This allows, for example, to assist a surgeon operating remotely by preventing the accidental penetration of critical tissue.

Haptics is an area where the computational requirement associated with the resolution of the dynamics and contact forces of the virtual environment in real time is particularly challenging. The human sense of touch is extremely susceptible to slight vibrations. Therefore the required computations need to be performed fast enough to control the haptic device at rates of $1000 \mathrm{~Hz}$. The collision detection methods we developed employ bounding sphere hierarchies to address complex environments, modeled by many thousands of polygons. The algorithms to compute the dynamic motions including the resolution of contact forces are described in the next section.

We have developed methods to facilitate the haptic display of complex environments. The fidelity of the haptic sensation and the robustness of the interaction with the environment is achieved by using the concept of a virtual proxy to represent the user's physical probe or finger. The virtual proxy can be viewed as if connected to the user's finger by a stiff spring. The haptic device is used to generate the forces of the virtual spring which appears to the user as the constraint forces caused by contact with a real environment. This framework also allows haptic shading to smooth the edges in polygon data and to display surface properties, such as friction and texture.

\section{Dynamic Simulation}

We developed a general framework [12] for the resolution of multi-contact between articulated multi-body systems in the context of operational space control for robots [7]. Using this framework, the dynamic relationships between all existing contact points can be described. This relationship is characterized by the masses as perceived at the contact points. A force exerted at a contact point, be it from a collision with another object or from the interaction with a user, can be translated into forces at all related contact points. The necessary computations can be performed with an efficient recursive algorithm.

The contact space representation allows the interaction between groups of dynamic systems to be described easily without having to examine the complex equations of motion of each individual system. As such, a collision model can be developed with the same ease as if one was considering interaction only between simple bodies. Impact and contact forces between interacting bodies can then be efficiently solved.

For the simulation of articulated body dynamics moving in free space, our effort was aimed at algorithms for robotic mechanisms with branching structures in order to address the complexities of the applications outlined above. Building on previous work in this area, we developed a recursive algorithm for computing the operational space dynamics and control of an n-joint branching redundant articulated robotic mechanism with $\mathrm{m}$ operational points $[2,3]$. An operational point is a point on the robot at which a certain behavior is controlled; in most cases such a point represents an end effector (see also Section 4). The computational complexity of this algorithm is $O\left(n m+m^{3}\right)$, while existing symbolic methods required a computational effort of $O\left(n^{3}+m^{3}\right)$. Since m can be considered as a small constant in practice, this algorithm attains a linear time $O(n)$ as the number of links increases.

These framework for contact resolution and dynamic simulation were integrated with our haptic rendering system [12] to provide a general environment for interactive haptic dynamic simulation. Figure 2 illustrates a virtual environment that has been modeled with this system. Two humanoid figures are colliding and interacting with numerous objects in the environment. 


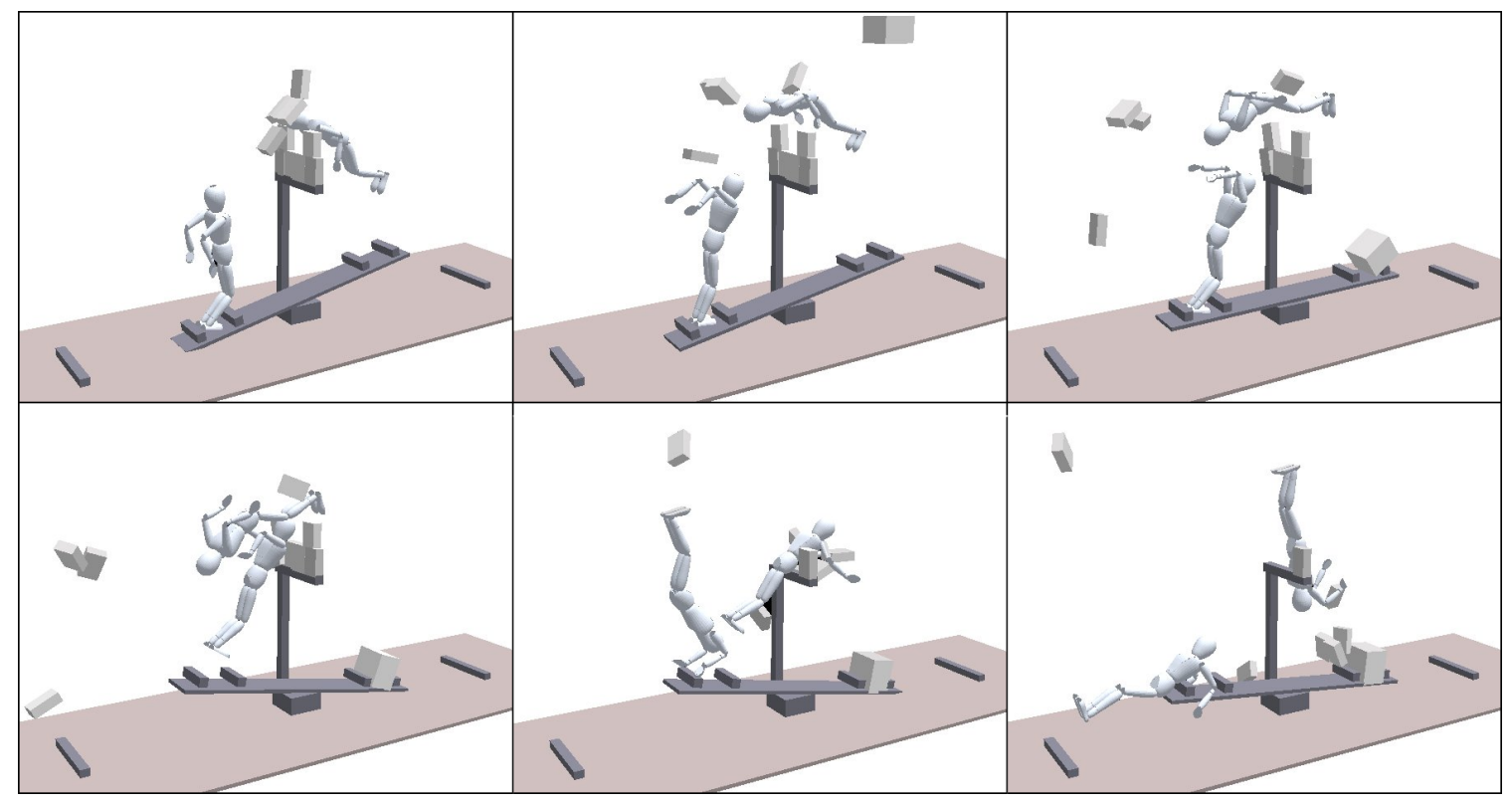

Figure 2: This sequence of images shows the complicated dynamic interaction between two humanoid figures and a large number of objects in the scene. The many contacts between objects in this scene and their resulting motions are computed interactively in accordance with their dynamic properties.

\section{Whole-Robot Control: Task and Posture}

For robots with human-like structures tasks are not limited to the specification of the position and orientation of a single effector, or operational point. For these robots, task descriptions may involve combinations of coordinates associated with one or both arms, the head-camera, and/or the torso. The remaining freedom of motion is assigned to various criteria related to the robot's posture and its internal and environmental constraints in the form of posture energies.

Conventionally robot dynamics are described in terms of the robot joint motion. The operational space formulation [7] provides an effective framework for dynamic modeling and control of branching mechanisms in terms of their operational points or end effectors. As a consequence, the desired behavior at an operational point can be described directly in terms of its motion, rather than in terms of the joints causing its motion. In other words, the operational space framework allows the direct control of the task, implicitly accounting for the dynamics and kinematics associated with the manipulator.

A generalized torque/force relationship $[7,8]$ provides a decomposition of the total joint torque command acting on the robot into two dynamically decoupled command torque vectors: the torque corresponding to the task behavior and the torque that only affects posture behavior. The former is used to command motions at the operational points of the robot, whereas the latter performs motion using the redundant degrees of freedom of the robot with out affecting task behavior. This framework extends very easily to robots with branching structures of $m$ effectors or operational points.

Dynamic consistency is the essential property for the task behavior to maintain its responsiveness and to be dynamically decoupled from the posture be- 

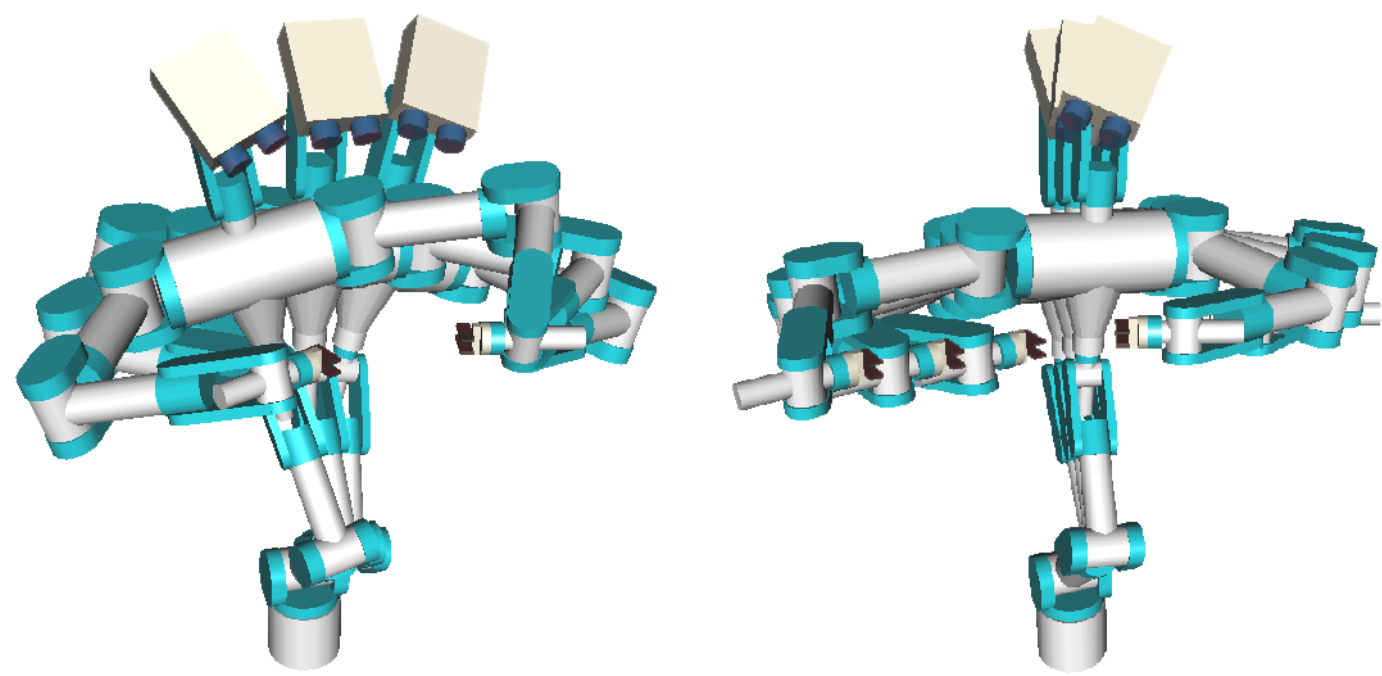

Figure 3: Dynamic consistency and posture behavior: a sequence of snapshots from the dynamic simulation of a 24-degree-of-freedom humanoid system. On the left, the task is to maintain a constant position for the two hands, while achieving hand-eye coordination. The posture motion has no effect on the task. On the right, the task also involves hand-eye coordination and motion of the common hand position. This position is interactively driven by the user. The posture is to maintain the robot total center-of-mass along the z-axis.

havior. This is illustrated in Figure 3 (left), where a robot (a 24-degree-of-freedom humanoid system) was commanded to keep the position of both hands constant (task behavior) while moving its left and right arm (posture behavior). Notice that dynamic consistency enables task behavior and posture behavior to be specified independently of each other, providing an intuitive control of complex systems. In Figure 3 (right) the robot posture can be controlled to maintain the robot total center-of-mass aligned along the z-axis of the reference frame. This resulting behavior is task execution at the operational points, while redundant degrees of freedom are used to maintain balance constraints to prevent tipping. More complex posture behaviors can be obtained by combination of simpler behaviors. We are currently exploring the generation of human-like natural motion from motion capture of human and the extraction of motion characteristics using human biomechanical models.

In addition to control methods to generate task and posture behavior for a single robot, effective cooperation strategies are needed both for the cooperation of multiple robots and for the interaction between robots and humans. Several cooperative robots, for instance, may support a load while being guided by the human to an attachment, or visually following the guide to a destination. Our approach to these problems is based on the integration of virtual linkage [9] and the augmented object model. The virtual linkage characterizes internal forces, while the augmented object describes the system's closed-chain dynamics. This approach has been successfully implemented on the Stanford robotic platforms for cooperative manipulation and human-guided motions.

\section{$5 \quad$ Elastic Plans}

A robotic system must be capable of sufficient level of competence to avoid obstacles during motion. Even when a path is provided by a human or automatic 


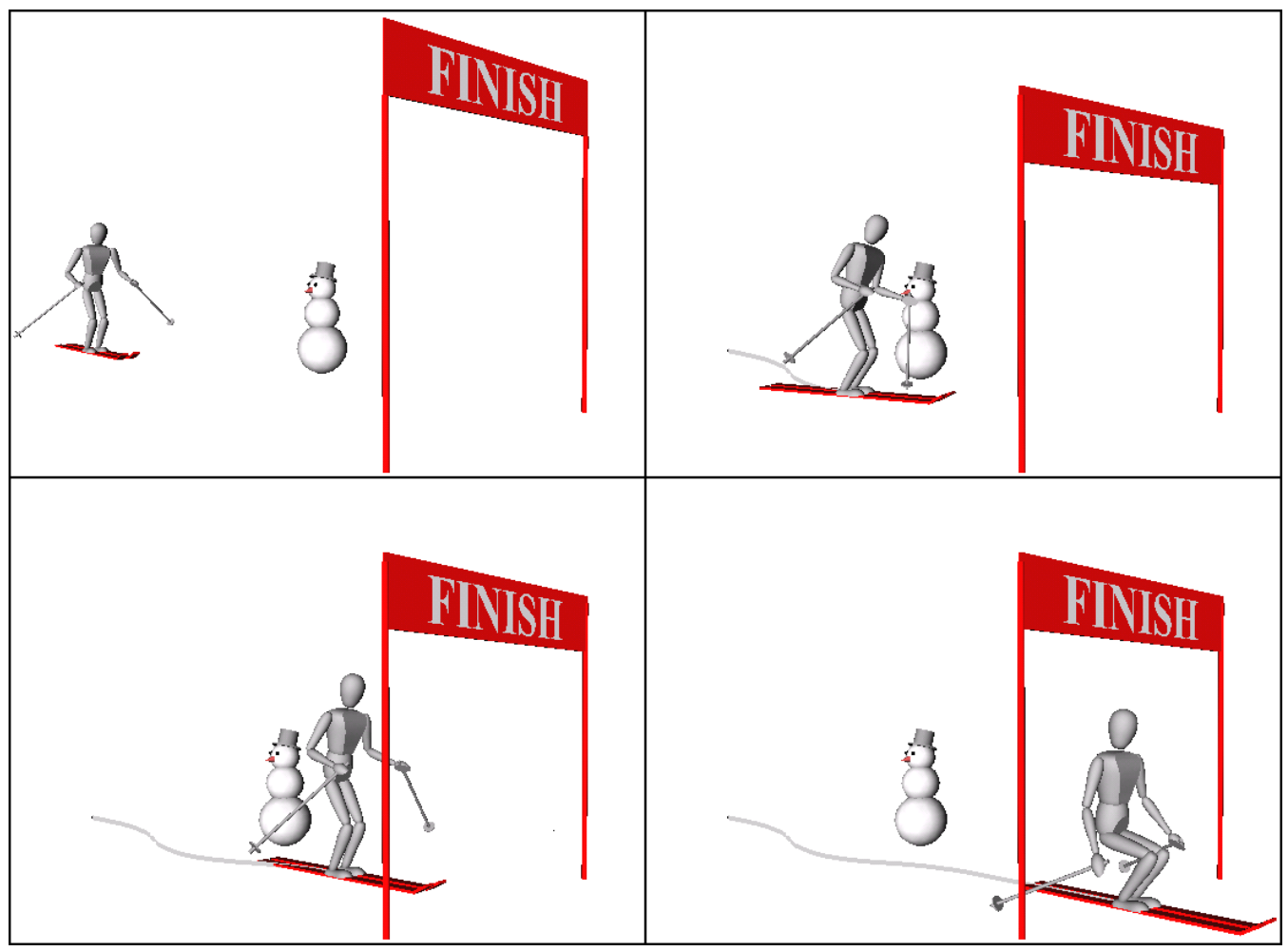

Figure 4: The trajectory of the humanoid figure is modified in real time as the snowman moves into its path and the finish banner is being lowered. Note how the ski poles are moved closer to the body to avoid the snowman and how a natural posture is maintained while passing under the finish banner.

planner, sensor uncertainties and unexpected obstacles can make the motion impossible to complete. Our research on the artificial potential field method [6] has addressed this problem at the control level to provide efficient real-time collision avoidance. Due to its local nature, however, reactive methods are limited in their ability to deal with complex environments. Our investigation of a framework to integrate real-time collision avoidance capabilities with a global collision-free path has resulted in the elastic strip approach [1], which combines the benefits of global planning and reactive systems in the execution of motion tasks.

The elastic strip framework modifies a specified path in real time to accommodate potential interac- tions with other robots or objects in the environment. This enables goal-directed motion in environments that change unpredictably. Due to the fact that the entire path is modified, the problem of local minima exhibited by purely local methods, is avoided. In order to satisfy the real-time requirements of the targeted applications, the efficiency of the elastic strip framework is of great importance. Real-time performance is achieved by the use of efficient free space computation and representation techniques.

The elastic strip framework exploits the decomposition of a robot's motion into task and posture, as described in Section 4, to enable task-consistent realtime path modification. This allows robots to perform desired behavior without interrupting task ex- 
ecution. The overall behavior can consist of a combination of various simple behaviors, such as maintaining a desired posture or avoiding collisions with obstacles. When the physical limitations of the robot render the simultaneous execution of task and additional behavior inconsistent, task execution can be automatically suspended; it is resumed when taskconsistency can be achieved.

An example of a real-time path modification in interaction with the environment is shown in Figure 4 , where a skiing humanoid avoids a moving snowman and crouches under the lowering finish banner. The snowman is avoided by performing a detour, but also by moving the ski pole closer to the body. The crouching behavior of the skier as it passes under the banner is the result of posture control. The ability to combine task execution with obstacle avoidance and posture behavior, as well as the ability to suspend and resume tasks, provide an important foundation for complex mechanical systems to operate autonomously in virtual or real worlds.

\section{Conclusion}

Advances toward the challenge of robotics in human environments depend on the development of the basic capabilities needed for both autonomous operations and human/robot interaction. In this article, we have presented methodologies for whole-robot coordination and control, cooperation between multiple robots, interactive haptic simulation with contact, dynamic simulation and real-time modification of collision-free path to accommodate changes in the environment. These developments provide some of the basic foundations in the effort to create robots with the advanced capabilities needed for human environments. The real-time capability shared among these developments is a key characteristic in providing the computational tools for a variety of applications at the intersection of the physical and the virtual world.

\section{References}

[1] Oliver Brock and Oussama Khatib. Elastic strips: Real-time path modification for mobile manipulation. In Proceedings of the International Symposium of Robotics Research, pages 117-122. Springer Verlag, 1997.

[2] Kyong-Sok Chang and Oussama Khatib. Operational space dynamics: Efficient algorithms for modellinga nd control of branching mechanisms. In Proceedings of the International Conference on Robotics and Automation, pages 850856, San Francisco, USA, 2000.

[3] Roy Featherstone. Robot Dynamics Algorithms. Kluwer Academic Publishers, 1987.

[4] P. W. Finn and Lydia E. Kavraki. Computational approaches to drug design. Algorithmica, 25:347-371, 1999.

[5] K. Hirai, M Hirose, Y. Haikawa, and T. Takenaka. The development of Honda humanoid robot. In Proceedings of the International Conference on Robotics and Automation, volume 2, pages 1321-1326, Leuven, Belgium, 1998.

[6] Oussama Khatib. Real-time obstacle avoidance for manipulators and mobile robots. International Journal of Robotics Research, 5(1):90-98, 1986.

[7] Oussama Khatib. A unified approach to motion and force control of robot manipulators: The operational space formulation. International Journal of Robotics and Automation, 3(1):43-53, 1987.

[8] Oussama Khatib. Inertial properties in robotics manipulation: An object-level framework. International Journal of Robotics Research, 14(1):1936, 1995.

[9] Oussama Khatib, Kazu Yokoi, Kyong-Sok Chang, Diego Ruspini, Robert Holmberg, and Arancha Casal. Coordination and decentralized cooperation of multiple mobile manipulators. Journal of Robotic Systems, 13(11):755764, 1996. 
[10] Jean-Claude Latombe. Motion planning: A journey of robots, molecules, digital actors, and other artifacts. International Journal of Robotics Research, 18(11):1119-1128, 1999.

[11] Koichi Nishiwaki, Tomomichi Sugihara, Satoshi Kagami, Fumio Kanehiro, Masayuki Inaba, and Hirochika Inoue. Design and development of research platform for perception-action integration in humanoid robot : H6. In Proceedings of the International Conference on Intelligent Robots and Systems, volume 3, pages 1559-1564, 2000.

[12] Diego C. Ruspini and Oussama Khatib. Collision/contact models for dynamic simulation and haptic in teraction. In 9th International Symposium of Robotics Research (ISRR'99), pages 185-195, Snowbird, Utah, U.S.A., October 1999. 